\title{
Relación Entre Biomasa Aérea Y Producción Primaria Neta Con Las Variaciones Estacionales De Precipitaciones Y Temperaturas En Pastizales Del Sur De La Provincia De Córdoba
}

\author{
Cerutti, A., N. Mónaco \\ M. J. Rosa y V. Santa. \\ Departamento de Ecología Agraria. Facultad de Agronomía y Veterinaria. \\ Universidad Nacional de Río Cuarto, Argentina.
}

doi: 10.19044/esj.2017.v13n5p117 URL:http://dx.doi.org/10.19044/esj.2017.v13n5p117

\begin{abstract}
Natural grasslands of the planet are very important reservoirs of flora and fauna that provide various ecosystem services benefits. To maintain and improve their condition, it is necessary to study them and understand their composition and functioning as well as their evolution over time. In the present work the influence of climatic variables, temperature and rainfall on the aerial biomass behavior and the net primary productivity of a pasture were analyzed. For this purpose, a natural pasture was located on the banks of the Chocancharava River, in the town of Río Cuarto. Monthly sampling (September 2014 - August 2015) was carried out, cutting aerial biomass at ground level with tusar scissors and collecting biomass and mulch in 10 quadrants of $0.5 \times 0.5 \mathrm{~m}(0.25 \mathrm{~m} 2)$ and dried in stove to constant weight. This material was separated into compartments: green (BV), dry (BS) and mulch (BM) to determined the available biomass. For statistical analysis, a completely randomized design (DCA) of fixed effects with Infostat program was used. The highest contribution of BV was in summer months, $4262 \mathrm{~kg} /$ ha of $8772 \mathrm{~kg} /$ ha corresponding to the total cycle. The highest BS was determined in winter with $2715 \mathrm{~kg}$ / ha of the total $6663 \mathrm{~kg} / \mathrm{ha}$. The litter presented a behavior similar to the BV, with the highest peaks in summer months $2478 \mathrm{~kg} /$ ha of $6004 \mathrm{~kg} / \mathrm{ha}$. The PPN presented a marked spring increase. Adding the positive increases in monthly PPN, a value of $1206.6 \mathrm{~kg}$ / ha * year was determined. Considering that higher temperatures and rainfall are recorded in summer (average $22^{\circ} \mathrm{C}$ and $422 \mathrm{~mm}$ ), it could be assert that these variables would explain a significant part of the variability in the total aerial biomass production of the pasture.
\end{abstract}


Keywords: Biomass, net primary productivity, climatic variables

\section{Resumen}

Los pastizales naturales del planeta constituyen importantes reservorios de flora y fauna proporcionando además diversos beneficios por los servicios ecosistémicos que prestan. Para mantener y mejorar la condición de los mismos es necesario estudiarlos y entender tanto su composición y funcionamiento como su evolución en el tiempo. En el presente trabajo se analizó la influencia de las variables climáticas, temperatura y precipitaciones en el comportamiento de la biomasa aérea y la productividad primaria neta de un pastizal. Para ello se realizó el relevamiento de un pastizal natural ubicado a las orillas del Río Chocancharava, en la localidad de Río Cuarto. A lo largo de un año se realizaron muestreos mensuales (Septiembre 2014 - Agosto 2015), cortando la biomasa aérea a ras de suelo con tijeras de tusar y recolectando mantillo en 10 cuadrantes de $0,5 \times 0,5 \mathrm{~m}\left(0,25 \mathrm{~m}^{2}\right)$, arrojados al azar. Este material se separó en compartimentos: verde (BV), seco (BS) y mantillo (BM), se secó en estufa hasta peso constante, determinando luego la biomasa disponible. Para el análisis estadístico se utilizó un diseño completamente aleatorizado (DCA) de efectos fijos con programa Infostat. El mayor aporte de BV fue en meses estivales, $4262 \mathrm{~kg} / \mathrm{ha}$ de $8772 \mathrm{~kg} / \mathrm{ha}$ correspondientes al total del ciclo, mientras que en BS se determinó en inverno con $2715 \mathrm{~kg} / \mathrm{ha}$ de los $6663 \mathrm{~kg} / \mathrm{ha}$ totales. El mantillo presento un comportamiento similar a la BV, con los mayores picos en meses estivales $2478 \mathrm{~kg} / \mathrm{ha}$ de $6004 \mathrm{~kg} / \mathrm{ha}$. La PPN presentó un marcado incremento primaveral. Sumando los incrementos positivos de PPN mensuales se determinó un valor de 1206,6 kgMS/ha*año. Teniendo en cuenta que las mayores temperaturas y precipitaciones se registran en verano (promedio $22^{\circ} \mathrm{C}$ y $422 \mathrm{~mm}$ ), se podría decir que estas variables explicarían una parte significativa de la variabilidad en la producción de biomasa total aérea del pastizal.

Palabras clave: Biomasa, productividad primaria neta, variables climáticas

\section{Introducción}

Los pastizales naturales comprenden aquellas regiones que se encuentran cubiertas por vegetación herbácea natural o seminatural, predominantemente gramíneas, con o sin plantas leñosas (Sala et al, 1986). Se considera pastizal natural a un área que produce forraje presentando una gran heterogeneidad en la estructura y composición de la vegetación, la cual surge de la combinación de los factores ambientales, tales como clima, suelo, topografía, factores bióticos como ausencia de monte, especies nativas adaptadas y diversos factores de manejo (Whittaker et al, 1973). 
Cubren una cuarta parte de la superficie del planeta, ayudando a mitigar el calentamiento global y evitan la erosión de los suelos, sin embargo son un ecosistema seriamente amenazado por la agricultura, la ganadería y la actividad forestal. Con sus 39 millones de kilómetros cuadrados en el mundo (casi una cuarta parte de la superficie terrestre continental), representan uno de los ecosistemas más extensos del planeta. El problema es que su conservación está seriamente amenazada porque en él están asentadas dos de las actividades productivas más importantes del mundo: la agroganadería y la industria forestal. De hecho, en estas regiones se produce gran parte de las semillas, la carne, la leche, la lana, el cuero y la madera que se consume en el mundo (D'Atri, 2007).

La superficie ocupada por los pastizales en Argentina comprende una amplia variedad de climas, suelos y vegetación. Ocupan aproximadamente un 70 \% del territorio nacional (Chiossone, 2011) del territorio continental, donde se desarrolla principalmente la cría bovina, ovina y caprina, cuyo principal componente de la alimentación son los pastizales naturales desarrollándose en sistemas de producción predominantemente extensivos, con desbalances estacionales entre oferta y demanda de forraje (INTA, 2011). Parte de los pastizales (30 mill. de has.) están constituidos por comunidades con montes y bosques nativos, en los que existe un desarrollo actual y potencial de sistemas de uso múltiple como los silvopastoriles (INTA, 2011).

Con respecto al manejo de los pastizales naturales, se deben incluir dos conceptos básicos, el primero en relación a la producción ganadera que otorga sustentabilidad económica a las empresas rurales y el segundo, en relación a la conservación del recurso natural (pastizal) que permite la continuidad de esta producción en el tiempo (Aguilera et al, 1999).

\section{Objetivos}

-Evaluar la evolución de la biomasa disponible a lo largo de un año en el pastizal natural y su variación estacional en función de las temperaturas y precipitaciones.

-Estudiar los cambios estacionales en la producción primaria neta en el pastizal natural en función de las temperaturas y precipitaciones.

\section{Materiales y métodos}

\section{Descripción del área de estudio}

El presente estudio se desarrolló durante el periodo comprendido entre octubre 2014 y septiembre 2015, en un pastizal natural ubicado a las orillas del Río Chocancharava en en áreas aledañas a la Universidad

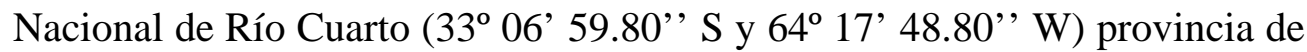
Córdoba, República Argentina (Figura N¹). 


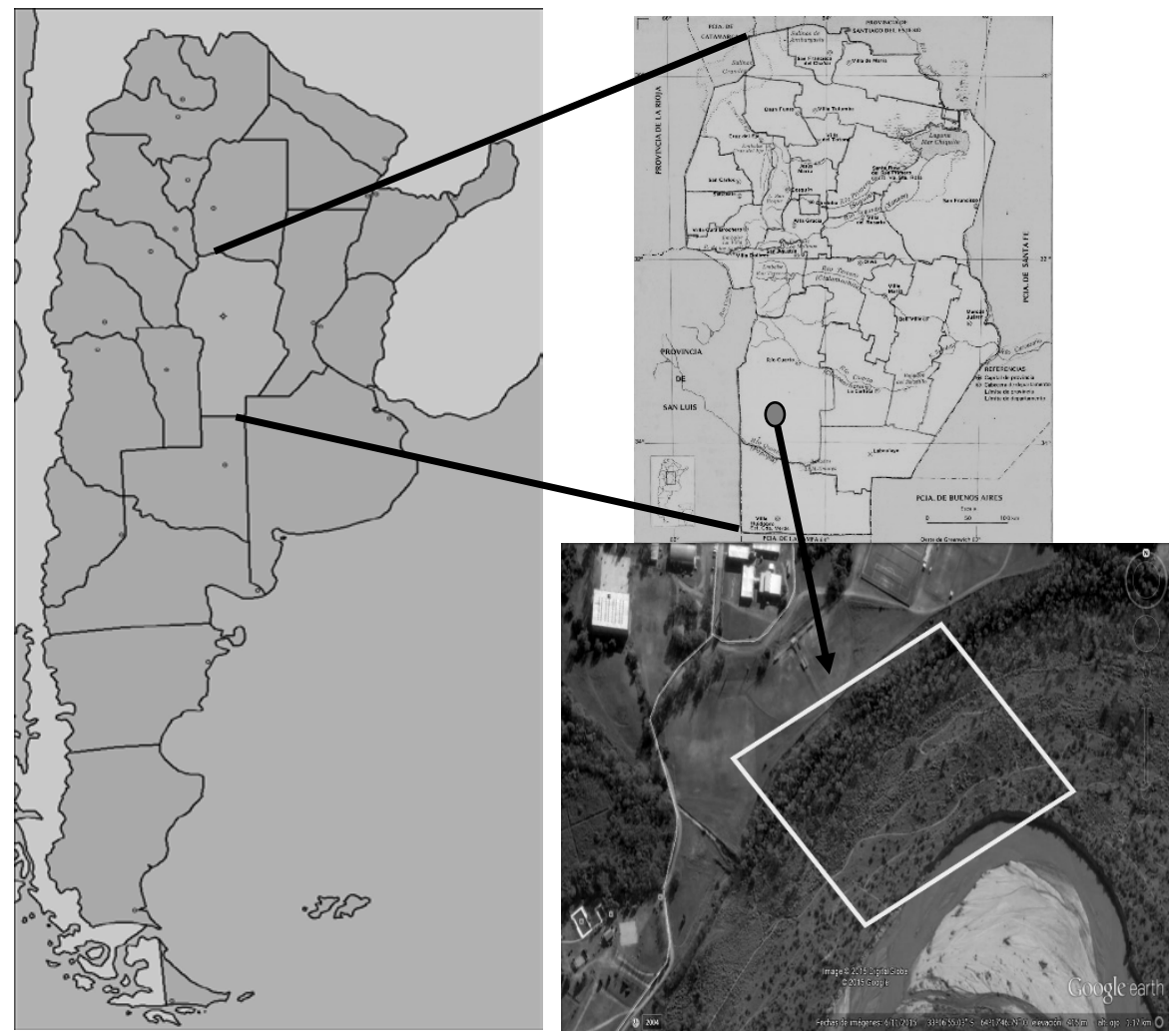

Figura N¹: Localización del área de estudio

\section{Caracterización edáfica}

El loess, material originario de estos suelos, posee un porcentaje elevado de limos y es rico en carbonato de calcio. Estos caracteres del material, sumados a las condiciones climáticas de una planicie subhúmeda a semiárida y la vegetación natural bajo la cual evolucionaron, confieren a los suelos las características más sobresalientes que condicionan su utilización y definen sus potencialidades (Jarsun, 1993).

Los Haplustoles son los suelos dominantes de la región, se caracterizan por ser altamente productivos, profundos, bien drenados, fértiles, con un horizonte superficial rico en materia orgánica y con el complejo de cambio dominado por el calcio teniendo una buena estructura. Sin embargo, el alto contenido en limo les confiere cierta fragilidad e inestabilidad estructural, que se manifiesta por una tendencia al encostramiento y al "planchado", punto inicial de los escurrimientos y de los procesos erosivos (Jarsun, 1993). 
- Analizando más puntualmente el área bajo estudio se trata de un Haplustol fluvéntico, de textura franca gruesa. Es un suelo bien drenado, desarrollado a partir de sedimentos fluviales de texturas contrastantes francas, franco limosas y franco arcillo limosas. Se caracteriza por poseer capas en profundidad o subsuperficiales donde aumenta abruptamente la materia orgánica. Ocupa las terrazas del Río Cuarto y derrames altos suavemente ondulados en las proximidades de la ciudad de $\mathrm{La}$ Carlota, provincia de Córdoba (INTA, 1993).

\section{Caracterización climatológica}

El área bajo estudio está ubicada en el dominio climático semihúmedo, con un régimen pluviométrico tipo monzónico, concentrando más del $80 \%$ de las lluvias en el semestre cálido. La precipitación media anual es de 782,5 mm (Serie 1981 - 2010) (Servicio de agrometeorología. UNRC).

El régimen térmico es templado con una temperatura media del mes más cálido (Enero) de $22,7^{\circ} \mathrm{C}$ y $9,4^{\circ} \mathrm{C}$ la del mes más frío (Julio), con una media anual para la serie de $16,3^{\circ} \mathrm{C}$ (Servicio de agrometeorología. UNRC).

- La fecha media de la primera helada meteorológica es el 25 de mayo ( $\pm 14,3$ días) y la fecha media de la última helada es el 12 de septiembre ( $\pm 20,3$ días). Esto indica que el período libre de helada alcanza una amplitud media de 256 días (Seiler et al., 1995).

- Los vientos prevalecen frecuentemente del $\mathrm{N}$ y NE y su mayor velocidad se registra durante el periodo comprendido entre fines de agosto y octubre (Fabricius y Rotondo, 1993). El granizo es un fenómeno que se presenta normalmente en el período estival.

\section{Caracterización de la vegetación}

La provincia de Córdoba (República Argentina) forma parte de las Provincias Fitogeográficas: Chaqueña, del Espinal y Pampeana, de Norte a Sur (Cabrera, 1976).

El pastizal analizado está ubicado en la Provincia Fitogeográfica del Espinal. El tipo de vegetación dominante es el bosque xerófilo. Caracteriza esta provincia la dominancia de especies arbóreas del género Prosopis, acompañadas por otros árboles. El lugar de estudio se ubica en el Distrito del 
Algarrobo (Cabrera, 1976). La comunidad climax es el bosque de algarrobo, unas veces algarrobo negro (Prosopis nigra), otras algarrobo blanco (Prosopis alba) acompañados por el tala (Celtis spinosa), el chañar (Geoffroea decorticans), y otros árboles característicos de la provincia (Cabrera, 1976).

En los sitios en los que las actividades agrícolas han sido abandonadas se presentan pastizales dominados generalmente por especies de la región pampeana. Dentro de estos parches de vegetación herbácea se encuentra chloris (Chloris cilata), gramon (Cynodon dactylon), linaria (Linaria vulgaris), pasto cuaresma (Digitaria sanguinalis), pasto horqueta (Paspalum notatum), gramilla blanca (Paspalum vaginatum), llanten (Plantago lanceolada), pasto plomo (Gamochaeta filaginea), pasto ilusión (Eragrostis lugens) y poa (Poa ligularis) (Poloni, 2012).

\section{Metodología}

Se caracterizó el pastizal natural según sus recursos abióticos (precipitaciones, temperaturas y características edáficas) y sus recursos bióticos (especies nativas e introducidas). Los datos referidos a precipitaciones y temperaturas fueron aportados por la cátedra de Agrometeorología (UNRC) para el período en estudio y también se obtuvieron promedios históricos para analizar el comportamiento de estas dos variables (periodo 1981-2010). La caracterización edáfica se obtuvo a partir del Atlas de Suelos de la República Argentina y además se realizó una calicata en el lugar de estudio. La vegetación nativa se caracterizó a partir de la información de las Regiones Fitogeográficas Argentinas (Cabrera, 1976).

La recolección de datos se realizó mensualmente sobre parches de vegetación herbácea, dejando de lado especies arbustivas, subarbustivas y arbóreas (Monaco et al., 2015). En cada fecha de muestreo, se tomaron 10 muestras en sitios al azar, con cuadrantes de $0,5 \times 0,5 \mathrm{~m}\left(0,25 \mathrm{~m}^{2}\right)$.

La disponibilidad de biomasa se determinó según la metodología tradicional, cortes a ras del suelo en cada cuadrante con tijera de tusar, luego se separó este material en compartimentos verde (BV), seco (BS) y mantillo (BM) para llevar a estufa hasta peso constante y determinar así la biomasa disponible (kg/ha) (Fernandez, 2004).

Con los datos de BV obtenidos en cada corte y el tiempo transcurrido entre corte y corte se calculó la Producción Primaria Neta (PPN), según la siguiente fórmula (Milner y Hughes, 1968):

PPN = (BV2-BV1) / (T2-T1)

\section{Donde:}

PPN= Producción Primaria Neta Aérea.

$\mathrm{BV} 2=$ biomasa verde final. 
BV1= biomasa verde inicial.

T2- T1= días transcurridos entre cada uno de los períodos de muestreo.

Los resultados para biomasa y PPN (kg/ha*año) se expresan mensual y estacionalmente. Para el análisis estadístico de las variables se utilizó un diseño completamente aleatorizado (DCA) de efectos fijos (Steel y Torrie, 1997), utilizando para ello el programa Infostat (Di Renzo et al., 2010).

\section{Resultados y discusión}

La Figura $N^{\circ} 2$ muestra las precipitaciones y las temperaturas medias mensuales históricas (1981-2010) y el comportamiento de estas mismas variables climáticas en el periodo en el que se realizó el ensayo. Esto se analizó con la finalidad de detectar diferencias y semejanzas entre el promedio histórico y las variables climáticas del período bajo estudio.

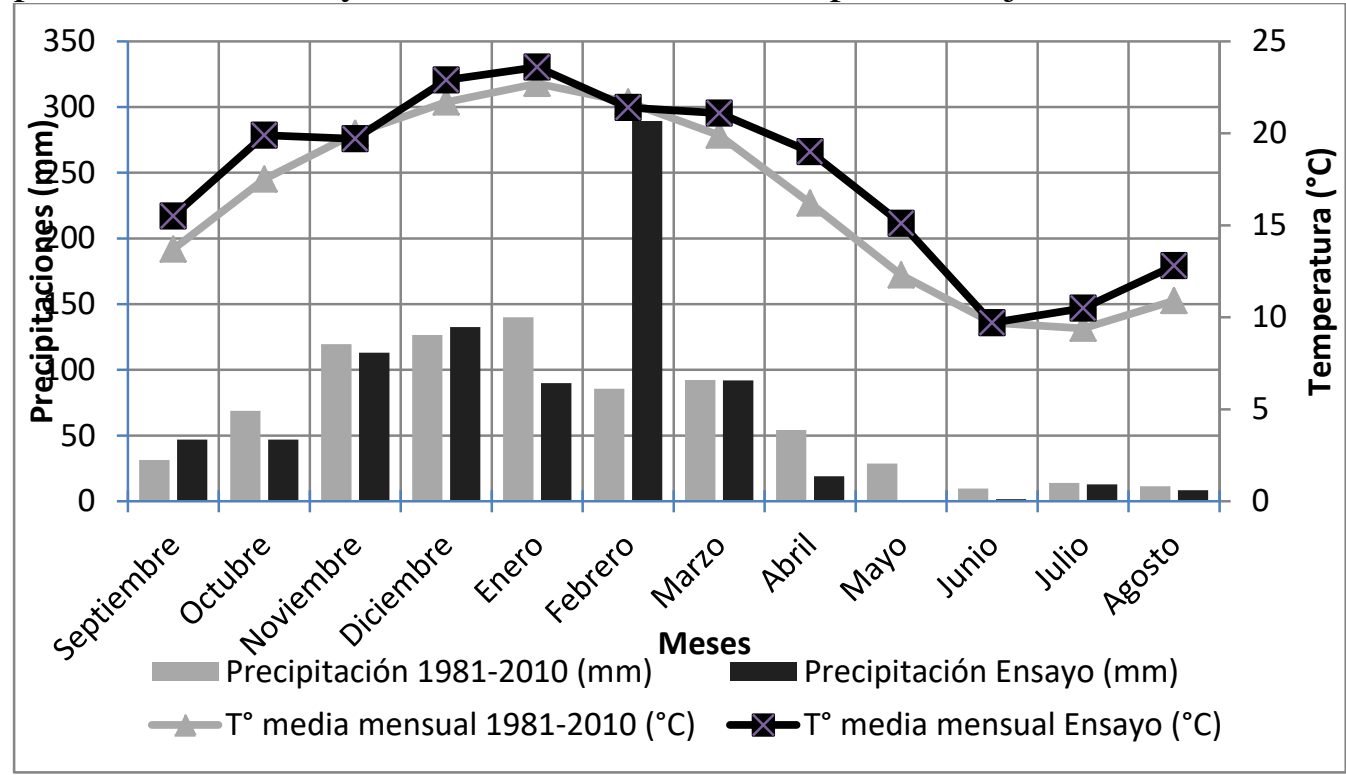

Figura $\mathrm{N}^{\circ} 2$ : Precipitaciones y temperaturas medias mensuales y medias mensuales históricas (periodo 1981-2010).

La precipitación anual registrada durante el período de realización del ensayo fue de 852,7 mm, presentando 70,5 mm sobre la media histórica. También, se dieron registros mensuales significativamente mayores a los promedios mensuales, destacando principalmente Febrero con una diferencia positiva de 203,8 mm. Por otro lado los menores registros con respecto a la media se dieron durante Enero, Abril, Mayo y Octubre. Los restantes meses tuvieron un comportamiento similar a los promedios mensuales.

Los registros de temperaturas medias mensuales obtenidas durante el ensayo se mantuvieron por encima de los valores históricos, destacándose los 
meses de Abril, Mayo y Octubre en los que la temperatura media mensual supero por más de $2,4^{\circ} \mathrm{C}$ a la media mensual histórica.

Teniendo en cuenta que los valores de BV, BS, BM y PPN fueron el objeto de estudio del presente trabajo se realizó a continuación un análisis de los mismos.

El comportamiento mensual de la BV, BS y BM en función de las precipitaciones se presenta en la Figura $\mathrm{N}^{\circ} 3$.

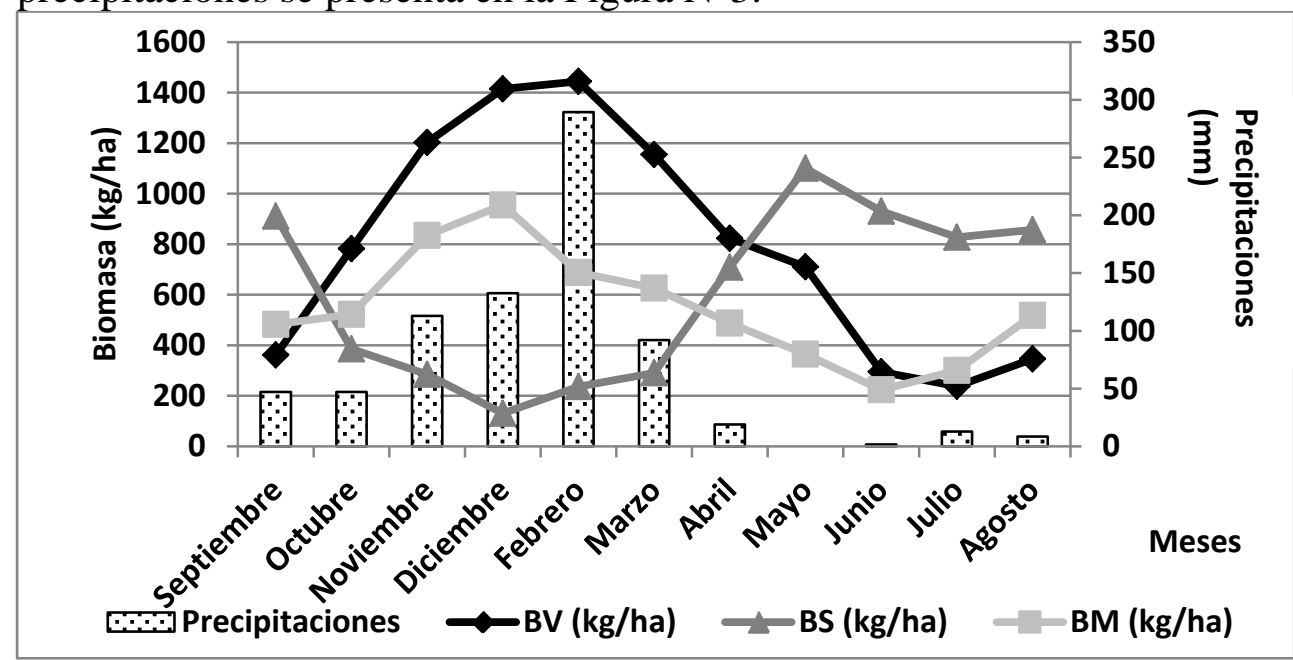

Figura N³: Comportamiento mensual de BV, BS y BM en función de las precipitaciones.

La producción total de BV fue de $8772 \mathrm{~kg} / \mathrm{ha}$ presentando una variación mensual significativa a lo largo del periodo bajo estudio (Tabla $\mathrm{N}^{\circ} 1$ ). Similar a lo encontrado por Menghi et al., 2000, en pastizales semejantes al estudiado, con los valores más altos en febrero y diciembre con el 32,6\% del total y los menores en julio, junio, agosto y septiembre $14,1 \%$.

Tabla N¹: Valores mensuales de BV (Test DGC). $\mathrm{p}<0.0001$.

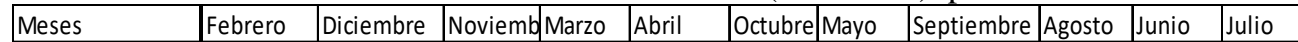

\begin{tabular}{|l|lll|l|l|l|l|l|l|l|l|}
\hline Medias (kgBV/ha) & $1444,6 \mathrm{a}$ & $1414,6 \mathrm{a}$ & $1202,8 \mathrm{~b}$ & $1154,8 \mathrm{~b}$ & $823,2 \mathrm{c}$ & $781,8 \mathrm{c}$ & $710,4 \mathrm{~d}$ & $361,6 \mathrm{e}$ & $345,6 \mathrm{e}$ & $294,4 \mathrm{e}$ & $238, \mathrm{e}$ \\
\hline
\end{tabular}

La diferencia en la producción de BV entre meses del año es estadísticamente significativa $(\mathrm{p}<0,05)$ probablemente debido a la variación de precipitaciones a lo largo del ciclo de estudio (Figura $\mathrm{N}^{\circ} 3$ ).

Con respecto a las estaciones, en la Tabla $\mathrm{N}^{\circ} 2$ se muestra que hay variaciones estacionales con valor y pendiente máxima en el verano (42\% del total anual). Mientras que en primavera y otoño se presentaron producciones intermedias y similares (49,4\% del total). Por otro lado, la BV de invierno equivale a un $8,6 \%$ de lo producido a lo largo de todo el ciclo. Estos valores son similares a los encontrados por Martin et al., 2009 y Wysiecki, 1993. Existen diferencias estadísticamente significativas $(\mathrm{p}<0.05)$ 
en lo que respecta a la producción de BV cuando se toman como referencia las estaciones (Tabla $\mathrm{N}^{\circ} 2$ ).

Tabla N²: BV según las estaciones. (Test DGC). p $=0.0117$

\begin{tabular}{|c|c|c|c|c|}
\hline Estación & Verano & Otoño & Primavera & Invierno \\
\hline Medias (kgBV/ha) & $1429,6 \mathrm{a}$ & $896,1 \mathrm{~b}$ & $782,1 \mathrm{~b}$ & $292,7 \mathrm{c}$ \\
\hline
\end{tabular}

La producción de BS presentó una fluctuación notable (Tabla $\mathrm{N}^{\circ} 3$ y Figura $\mathrm{N}^{\circ} 3$ ), el mayor valor se observó en mayo (16,5\%) y el menor en diciembre (2\%) de un total de $6663 \mathrm{~kg} / \mathrm{ha}$. La diferencia que existe en la producción de BS entre los meses del año es estadísticamente significativa $(\mathrm{p}<0,05)$ (Tabla $\left.\mathrm{N}^{\circ} 3\right)$ y se podrían atribuir las mismas al impacto de la variación de las precipitaciones.

Tabla N³: Valores mensuales de BS (Test DGC). $\mathrm{p}<0.0001$.

\begin{tabular}{|l|c|c|c|c|c|c|c|c|c|c|c|}
\hline Meses & Mayo & Junio & Septiembre & Agosto & Julio & Abril & Octubre & Marzo & Noviembre & Febrero & Diciembre \\
\hline Medias (kgBS/ha) & $1100,6 \mathrm{a}$ & $930,6 \mathrm{~b}$ & $910,6 \mathrm{~b}$ & $857,4 \mathrm{~b}$ & $827,3 \mathrm{~b}$ & $708,4 \mathrm{c}$ & $385,8 \mathrm{~d}$ & $291 \mathrm{~d}$ & $284,8 \mathrm{~d}$ & $236 \mathrm{~d}$ & $130,4 \mathrm{e}$ \\
\hline
\end{tabular}

$\mathrm{Al}$ realizar el análisis estacional de la BS (Tabla $\mathrm{N}^{\circ} 4$ ), en invierno se registró el valor más alto (38,2\% del total); en cuanto a la primavera y el otoño, tuvieron un comportamiento similar entre sí, sumando las dos estaciones 53,8\% de la BS anual. El verano aportó solamente el $8 \%$. Pese a esto no existen diferencias estadísticamente significativas en la producción estacional de BS.

Tabla N4: BS según las estaciones. (Test DGC). p=0.1399.

\begin{tabular}{|c|c|c|c|c|}
\hline Estación & Invierno & Otoño & Primavera & Verano \\
\hline Medias (kgBS/ha) & $871,8 \mathrm{a}$ & $700 \mathrm{a}$ & $527,1 \mathrm{a}$ & $183,2 \mathrm{a}$ \\
\hline
\end{tabular}

La biomasa mensual de mantillo presentó un comportamiento variable (Tabla $\mathrm{N}^{\circ}$ ), diciembre aportó la mayor cantidad (15,9\%) mientras que mayo, junio, julio sumaron, en conjunto un 14,8\% del total. La producción anual de BM fue de $6004 \mathrm{~kg} / \mathrm{h}$, superior a lo hallado por Menghi et al., 2000. La diferencia en la producción de mantillo entre los distintos meses del año es estadísticamente significativa $(\mathrm{p}<0,05)$ acompañando los cambios de precipitaciones en el ciclo bajo estudio.

Tabla N5: Valores mensuales de Mantillo (Test DGC). $\mathrm{p}<0.0001$.

\begin{tabular}{|l|c|c|c|c|c|c|c|c|c|c|c|}
\hline Meses & Diciembre & Noviembre & Febrero & Marzo & Octubre & Agosto & Abril & Septiembre & Mayo & Julio & Junio \\
\hline Medias(kgBM/ha) & $955,6 \mathrm{a}$ & $834,6 \mathrm{~b}$ & $687,8 \mathrm{c}$ & $626,4 \mathrm{c}$ & $522,2 \mathrm{~d}$ & $518,4 \mathrm{~d}$ & $487,6 \mathrm{~d}$ & $482,6 \mathrm{~d}$ & $364,6 \mathrm{e}$ & $300,4 \mathrm{e}$ & $224 \mathrm{e}$ \\
\hline
\end{tabular}

En cuanto a los cambios estacionales de BM (Tabla $\mathrm{N}^{\circ}$ 6) el verano y el invierno, representaron el 36,1\% y 15,3\% respectivamente del total anual, la primavera y el otoño presentaron valores intermedios entre los porcentajes anteriores, no existiendo diferencias estadísticamente significativas entre las estaciones. Menghi et al., 1998 encontraron picos de BM en primavera y otoño siendo éstos notables a diferencia del pastizal bajo estudio. 
Tabla N6: Mantillo según las estaciones. (Test DGC). p=0.0754.

\begin{tabular}{|c|c|c|c|c|}
\hline Estación & Verano & Primavera & Otoño & Invierno \\
\hline Medias (kgBM/ha) & $821,7 \mathrm{a}$ & $613,1 \mathrm{a}$ & $492,9 \mathrm{a}$ & $347,6 \mathrm{a}$ \\
\hline
\end{tabular}

En cuanto al régimen térmico, la variabilidad anual denoto situaciones de amplitud, principalmente en los meses otoñales con una temperatura mayor a la media histórica (Figura $\mathrm{N}^{\circ} 4$ ).

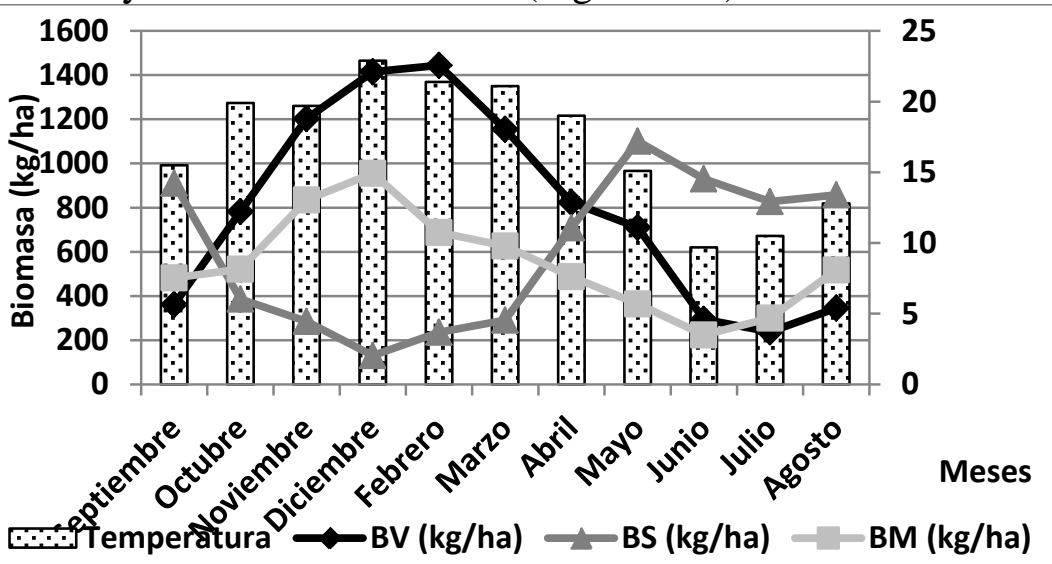

Figura $\mathrm{N}^{\circ}$ 4: Comportamiento de BV, BS y BM en función de temperaturas

Los resultados obtenidos indican un comienzo de ciclo con un incremento de $\mathrm{BV}$ (Figura $\mathrm{N}^{\circ} 4$ ) a partir de agosto que depende de la acumulación de temperatura previo al inicio de las lluvias. Menghi et al. (2000) y Lezana (2013) observaron comportamientos similares. El aumento de la BV continuó en los meses siguientes, alcanzando su pico más alto en febrero, comenzando a disminuir en marzo y alcanzando el mínimo valor en julio.

Es decir, la producción de BV tiene un comportamiento de campana. Esto coincide con lo encontrado por Arias Usandivaras, 2006, quien observó que la temperatura promueve el crecimiento del pastizal y la disminución de las precipitaciones lo limitan.

Analizando las fluctuaciones estacionales de la BS en función de la temperatura mensual (Figura $\mathrm{N}^{\circ} 4$ ) se observó que los incrementos de la temperatura mensual a partir de septiembre producen una disminución continua de la materia seca, hasta alcanzar el valor mínimo en diciembre con la máxima temperatura media mensual del ciclo bajo estudio. En los meses siguientes y con la reducción progresiva de la temperatura mensual, hay un aumento en la BS hasta que llega al máximo valor en el mes de mayo, previo al comienzo del invierno.

Cuando las precipitaciones o temperaturas comienzan a disminuir hay un incremento de la biomasa seca por la senescencia de especies primaveroestivales o el efecto de las heladas tempranas, situaciones muy normales en 
los meses otoñales. El pico que se da en otoño se debe a que una gran parte de la BV producida en los meses primavero estivales finaliza su ciclo vegetativo pasando al compartimiento de la BS.

El mayor aporte de mantillo (Figura $\mathrm{N}^{\circ} 4$ ) se realizó en diciembre $(955,6 \mathrm{~kg} / \mathrm{ha})$. Las condiciones ambientales de temperatura y precipitaciones al inicio del ciclo bajo estudio fueron favorables para el aumento del mantillo, dado que las especies que concluyen su ciclo pierden sus hojas y tallos y cubren la superficie. Sanchez (2009) encontró efectos similares sobre la biomasa seca, que pasaba a la hojarasca. La disminución de temperaturas y humedad características del avance de la estación otoñal provoca un decrecimiento en los valores de BM alcanzando el mínimo a finales de otoño en el mes de junio (224 kg/ha), no existiendo diferencias significativas entre las diferentes estaciones (Tabla $\mathrm{N}^{\circ} 6$ ).

Para determinar si las diferencias estadísticamente significativas obtenidas son atribuibles a la influencia de la temperatura y la precipitación se realizó el siguiente análisis de correlación. Los resultados se muestran a continuación.

Figura N5: Correlación entre Precipitaciones, Temperatura y BV.

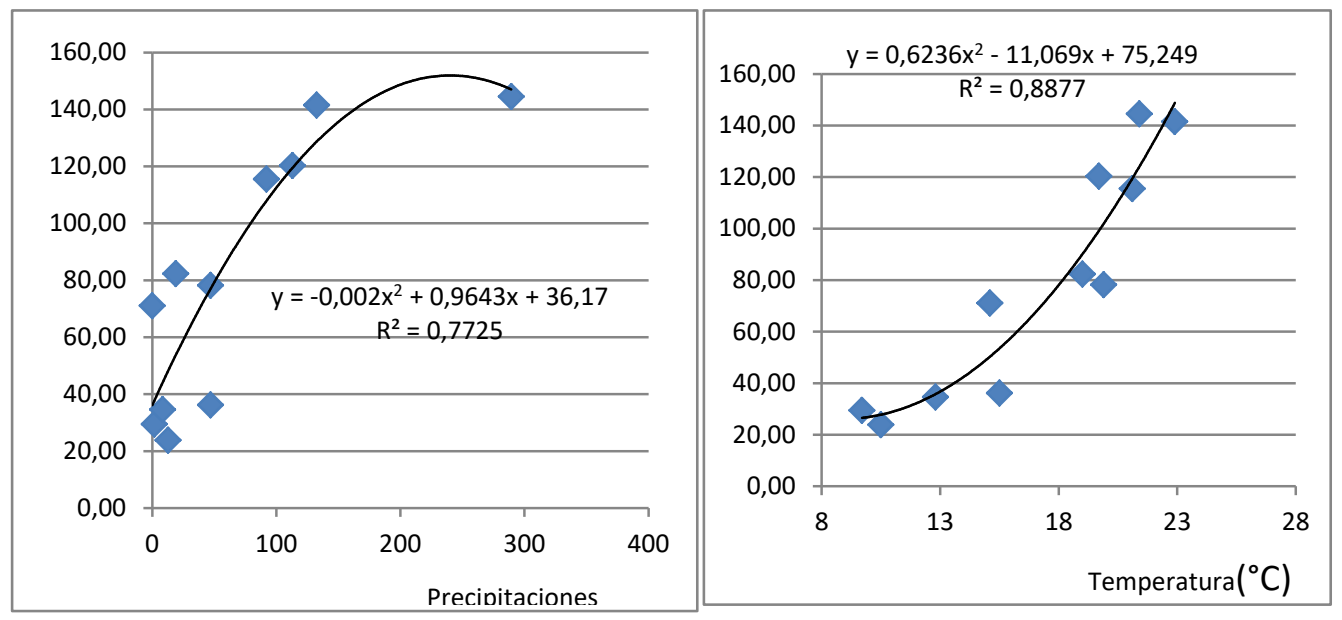

Como se observa en la Figura $\mathrm{N}^{\circ} 5$ hay una correlación positiva entre la producción de $\mathrm{BV}$, las precipitaciones y temperaturas dadas en el ciclo. Se halló que las precipitaciones explican el 77\% y las temperaturas un 88\% de la variabilidad de la producción de la BV. Esto demuestra que ambos factores climáticos tienen una influencia estadísticamente significativa en cuanto a la producción de este pastizal natural. Coincidiendo con lo encontrado por Arias, 2006; Martin et al., 2009 y Lezana, 2013.

La correlación entre las precipitaciones y las temperaturas con la BV que se halló en este trabajo es mucho mayor a la que obtuvo Menghi et al., 2000. Quizás esto se deba a que no existe aporte de una napa subterránea o 
de la escasa capacidad de retención hídrica del suelo sobre el que se encuentra el pastizal, lo cual hace a esta comunidad vegetal muy dependiente del aporte de las precipitaciones.

Como se observa en la Figura $\mathrm{N}^{\circ} 6$ existe una correlación negativa de la BS tanto con las precipitaciones como con la temperatura, por lo que a mayores precipitaciones y temperaturas menos valores de BS se producirán en el pastizal. Lo anterior es similar a lo encontrado por Sanchez et al., 2009.

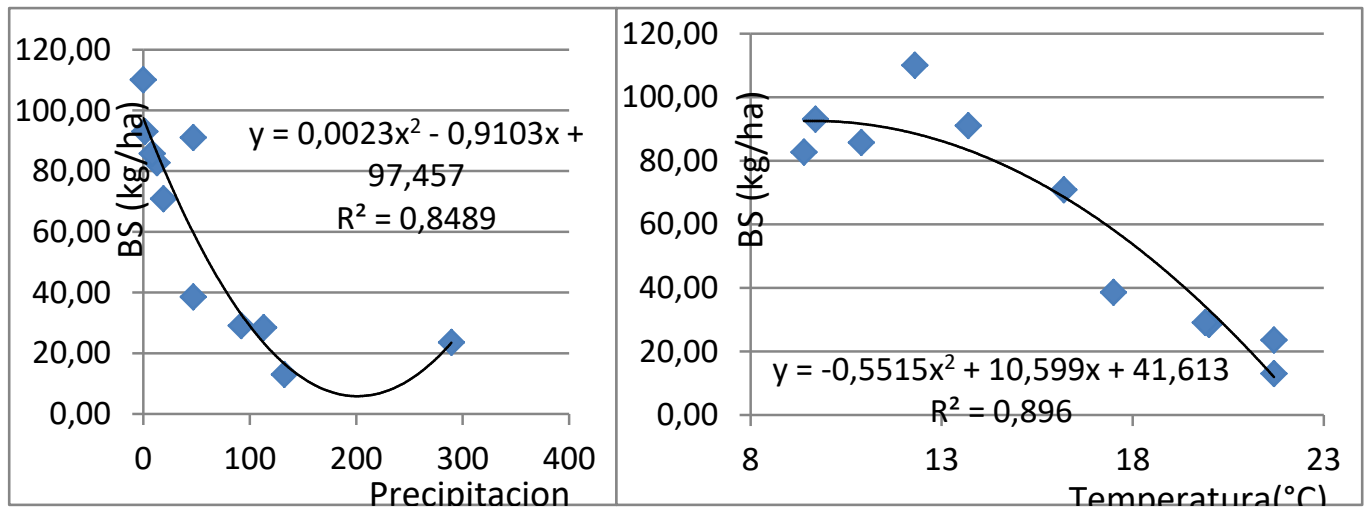

Figura $\mathrm{N}^{\circ}$ 6: Correlación entre Precipitaciones, Temperatura y BS.

El análisis de la correlación entre Precipitaciones, Temperatura y BM se muestra en la Figura $\mathrm{N}^{\circ} 7$.
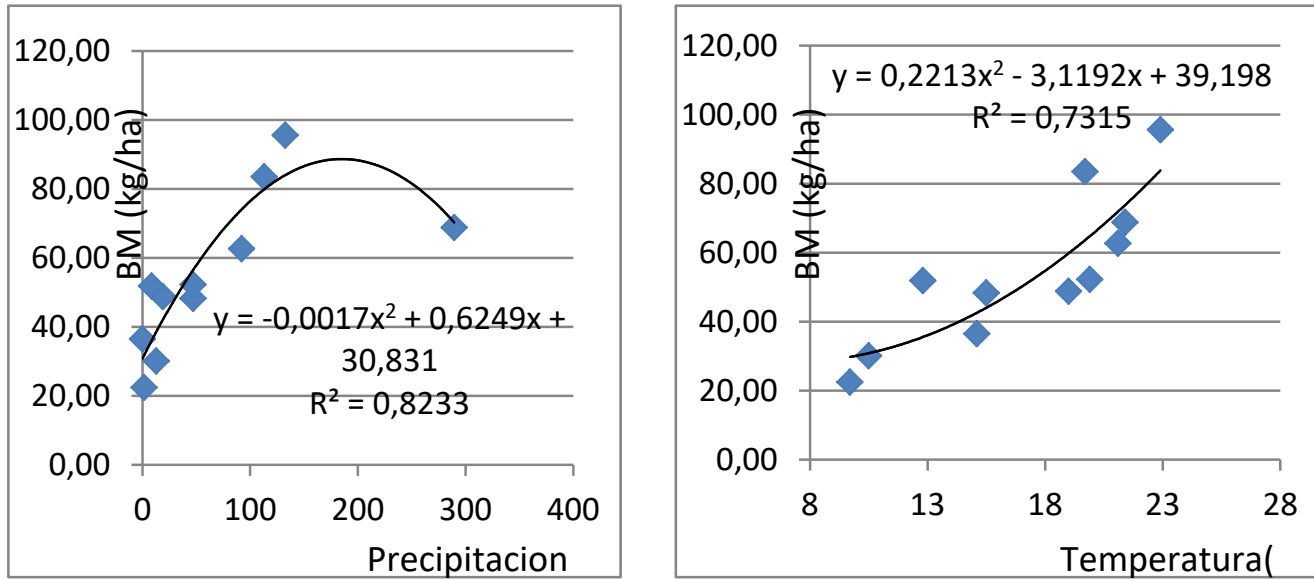

Figura $N^{\circ} 7$ : Correlación entre Precipitaciones, Temperatura y BS

Existe una correlación positiva entre las variables bajo estudio y el mantillo, es decir, que las precipitaciones y las temperaturas influyen en el comportamiento de la hojarasca. Para comprender el efecto de estos fenómenos sobre la estructura de la BS hay que tener en cuenta como lo anterior afecta al mantillo del pastizal. 
Se calculó la PPN correspondiente a cada uno de los meses y también la media estacional. En la Figura $\mathrm{N}^{\circ} 8$ se muestra el comportamiento de la PPN en relación a las precipitaciones.

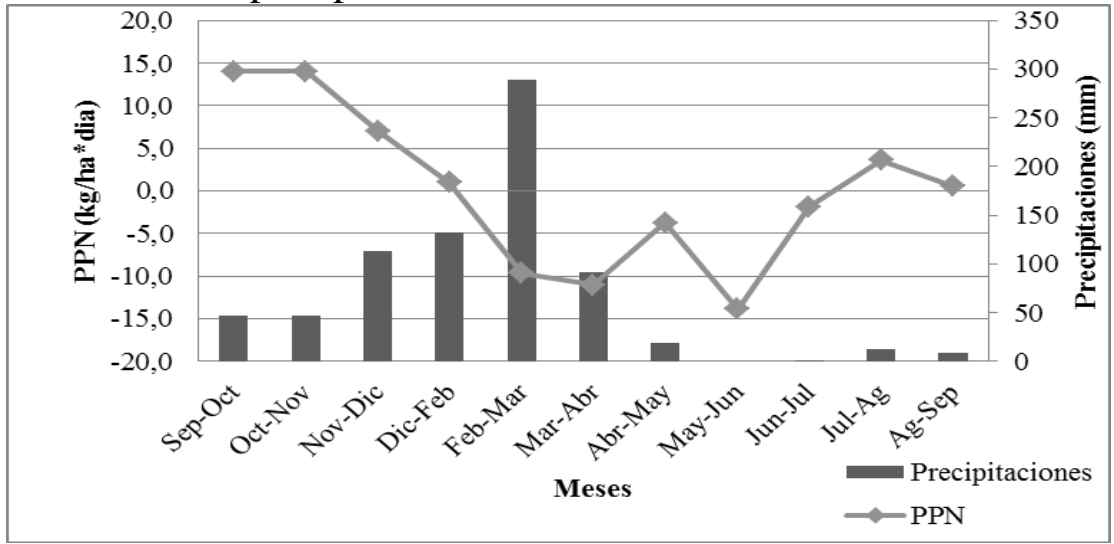

Figura $\mathrm{N}^{\circ}$ : Comportamiento de la PPN según precipitaciones del ensayo.

$\mathrm{Al}$ analizar la Figura $\mathrm{N}^{\circ} 8$ se percibe que no existe una relación clara entre las precipitaciones y la PPN. Esto queda demostrado en los últimos meses del verano, en los cuales a pesar de existir una considerable acumulación de agua en el perfil, hay una disminución de la PPN, y en los últimos meses invernales donde ocurre lo contrario hay un incremento en la productividad primaria neta.

En la Figura $\mathrm{N}^{\circ} 9$ se muestra el comportamiento de la PPN en relación a las temperaturas.

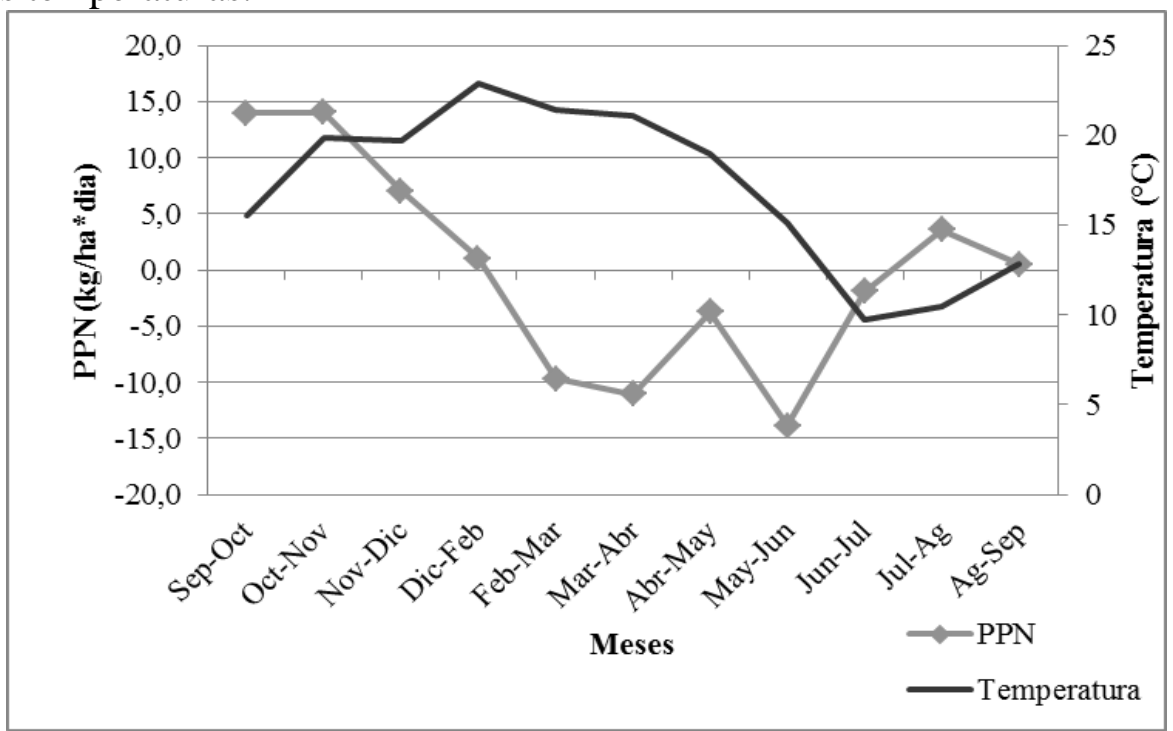

Figura N9: Comportamiento de la PPN según temperaturas del ensayo. 
A partir de los resultados obtenidos, se puede decir que la temperatura no tiene un impacto significativo sobre la PPN. Como se ve en la Figura $\mathrm{N}^{\circ}$ 9, a lo largo del ciclo bajo estudio, los meses en los que la temperatura fue mayor la productividad estuvo en un momento de transición hacia valores negativos, similar a lo ocurrido con las precipitaciones (Figura N8). Cuando la temperatura disminuyó en los meses otoñales la productividad primaria continuó descendiendo, alcanzando los mínimos valores a lo largo del ciclo. Cuando la temperatura comenzó a aumentar a fines del invierno y comienzos de la próxima primavera, la PPN comenzó a tener una tendencia positiva nuevamente.

Al analizar el comportamiento de la PPN, a lo largo del ciclo de crecimiento se observa que el pico de producción primaria se da en los meses de septiembre y octubre, coincidiendo con lo encontrado por Menghi et al., 2000. Lezana (2013) encontró valores altos en los mismos meses, pero obtuvo la máxima productividad en noviembre y enero. En los siguientes meses de este ensayo, comienza una disminución progresiva de los valores a lo largo de los meses estivales. Los menores valores se dan en febrero, marzo y mayo siendo estos meses junto con abril y junio los momentos en los cuales la productividad del pastizal es negativa. A partir de julio se reinició el ciclo de crecimiento del pastizal. Los investigadores antes mencionados también encontraron ese comportamiento en forma de campana, donde la mínima PPN se da en los meses otoño-invernales.

La producción primaria neta diaria fue variable según el mes promediando unos $6,7 \mathrm{~kg} / \mathrm{ha*}$ día, un valor menor a lo obtenido por Menghi et al. (2000) (7,7 a 25,7 kg/ha*día) y por Montani et al. (2000) (70 a 100 kg/ha*día). Fantino et al., 2010 obtuvieron un valor de PPN promedio de 32 kg/ha*día. Para un área de estudio similar, Rosa et al. (2009), obtuvieron una PPN de 6 a 7 kg/ha*día, dependiendo de las variables climáticas del ciclo de estudio.

El pastizal del ensayo tiene un marcado crecimiento primaveral. Wysiecki (1993) encontró que el pastizal que estudió tenía dos picos de crecimiento, uno en primavera y otro en otoño. Menghi et al. (2000) y Lezana (2013) encontraron picos de crecimiento en primavera y verano.

En el verano se dio una situación de transición donde la productividad paso a ser negativa significando esto que el pastizal produce menos BV de la que pasa a BS por diferentes procesos.

\section{Conclusión}

La estructura y dinámica de este pastizal depende de una interacción compleja de factores ente los cuales la precipitación y la temperatura explican una parte significativa de su variabilidad. 
Las temperaturas medias y las precipitaciones, tanto mensuales como estacionales, tienen una influencia estadísticamente significativa en la producción de BV. El inicio de la producción primavero-estival de BV se produjo independientemente del comienzo de las lluvias y estuvo condicionado en mayor medida por la temperatura. Por otro lado, las temperaturas y precipitaciones a nivel estacional no influyen en las fluctuaciones de BS y BM, pero si lo hacen los aportes mensuales de ambos.

A partir del análisis de las fluctuaciones de la PPN hay que mencionar que las variaciones pluviométricas y térmicas no explican de una manera significativa el comportamiento de la misma.

Los resultados que se obtuvieron en este trabajo muestran ciertas tendencias que podrían establecer las bases para entender el funcionamiento de los pastizales naturales.

\section{References:}

1. Aguilera, M.; Steinaker, D.; Demaría, M. y Giulietti, J. 1999. Guía Utilitaria De Manejo Para Pastizales Naturales Pampeanos Del Area Medanosa Central De Argentina. Información Técnica 153. INTA EEA, San Luis, Argentina.

2. Arias Usandivaras, L. 2006. Controles Climáticos De La Productividad Primaria De Pastizales De La Provincia De Corrientes. Trabajo Final De Graduación. Universidad De Buenos Aires, Facultad de Agronomía.

3. Cabrera, A. 1976. Las Regiones Fitogeográficas Argentinas. Enciclopedia Argentina De Agricultura, Jardinería Y Horticultura. Ed. ACME. Buenos Aires. Argentina. 2da. Edición. p. 85.

4. Chiossone, G. 2011. Pastizales Naturales De Argentina. Sitio Argentino De Producción Animal. EEA INTA El Colorado, Formosa.

5. D'Atri, P. 2007. Pastizales Del Mundo. Novedades de Biodiversidad en América Latina $\mathrm{N}^{\circ}$ 160. IPS (Inter Press Service) E IFEJ (Federación Internacional de Periodistas Ambientales).

6. Di Rienzo, J.; Casanovas, F.; Balzarini, M.; González, L.; Tablada, M.; Robledo, C. 2010. Infostat, Versión 2010, Grupo Infostat, FCA, Universidad Nacional de Córdoba, Argentina.

7. Fabricius, R. y Rotondo, V. 1993. Características Climáticas del Área Frutihortícola de Río Cuarto. En Diagnóstico y Evolución del Área Frutihortícola de Río Cuarto. Facultad de Agronomía y Veterinaria, Universidad Nacional de Río Cuarto. pp. 23-26.

8. Fantino, E.; Montani, N.; Monaco, N.; Bocco, O.; Rosa, MJ. y Heguiabehere, A. 2010. Efectos del pastoreo con diferentes tiempos de ocupación sobre los parámetros productivos de un pastizal (SE de Córdoba, Argentina central). Pastos XL(1), pp. 47-63. 
9. Fernández, H. 2004 Estimación de la Disponibilidad de Pasto. INTA. Estación Experimental Balcarce, Área de Producción Animal Balcarce, Bs. As. Argentina. p. 23.

10. INTA. 2011. Documento Base de Área Estratégica: Forrajes y Pasturas. Pergamino.

11. INTA y Gobierno de la Provincia de Córdoba. Plan Mapa de Suelos. 1993. Hoja 3363-27 La Carlota. Serie Carta de Suelos de la República Argentina. Escala 1:50.000. Córdoba.

12. Jarsun, B. 1993. Carta de Suelos de la República Argentina. Plan Mapa de Suelos. Hoja 3363-20 Ucacha. Inta-Agricultura, Ganadería Y Recursos Naturales. pp. 72. Córdoba (Argentina).

13. Lezana, L. 2013. Productividad Primaria Neta Aérea (PPNA) del Pastizal Natural en el Centro-Norte de Entre Ríos. INTA.

14. Martín, B.; Galleano, V.; Spiller, L.; Vilche, M. y Montico, S. 2009. Evaluación de la Productividad Primaria de un Pastizal Templado en Santa Fe, Argentina. Facultad de Ciencias Agrarias. Universidad Nacional de Rosario.

15. Menghi, M. 1998. Diversidad y Producción Primaria en un Pastizal Inundable no Pastoreado en ea Estepa Pampeana (Argentina Central). Pastos, Vol 28, T 1, pp. 51-67.

16. Menghi, M.; Seiler, R.; Montani, N.; Mónaco, N. y Rosa, M.J. 2000. Variación Anual e Interanual de la Producción de un Pastizal Inundable en la Estepa Pampeana (Argentina Central). Relación con la Precipitación y Temperatura. Centro de Ecología y Recursos Naturales Renovables. Facultad de Ciencias Exactas, Físicas y Naturales. Universidad Nacional de Córdoba. Ecología Vegetal Aplicada. Facultad de Agronomía y Veterinaria. Universidad Nacional de Río Cuarto.

17. Milner, C. y Hughes, R. 1968. Methods for the Measurement of the Primary Production of Grasslands. Blackweel Scientific Publi., 70. Oxford.

18. Mónaco, N.; Rosa, MJ.; Santa, V.; Autran, v: y Heguiabehere, a: 2015. Utilización de estimadores para determinación de biomasa a campo European Scientific Journal November 2015 edition vol.11, No.33 ISSN: 1857 - 7881 (Print) e - ISSN 1857- 7431

19. Montani, N.; Rosa, MJ.; Monaco, N., Menghi, M. y Magallanes Torres, S. 2000. Análisis de biomasa aérea y producción primaria en un pastizal natural inundable en clausura (La Felipa, Córdoba). $23^{\circ}$ Congreso de Producción Animal. Vol. 20 Sup.I, pp.233-234.

20. Poloni, G. 2012. Análisis de la Producción Primaria Medida y Estimada por Sensores Remotos en la Cuenca del Arroyo Chucul. 
Trabajo Final de Graduación. Facultad de Agronomía y Veterinaria. UNRC.

21. Rosa, MJ.; Monaco, N. y Monatani, N. 2009. Análisis de dos comunidades de pastizales naturales del sur-este de Córdoba sometidas a diferente manejo. Revista Universidad Nacional Río Cuarto 29 (1-2), pp. 3-18.

22. Sala, O.; Oesterheld, O.; León, R. y Soriano, A. 1986. Grazing effects upon plant community structure in subhumid grasslands of Argentina. Vegetation 67:27-32.

23. Sánchez, S; Crespo, G. y Hernández, M. 2009. Descomposición de la Hojarasca en un Sistema Silvopastoril de Panicum Maximum y Leucaena Leucocephala (Lam) de Wit Cv. Cunningham. II. Influencia de los Factores Climáticos. Estación Experimental de Pastos y Forrajes «Indio Hatuey». Central España Republicana, CP 44280, Matanzas, Cuba.

24. Seiler, R.; Fabricius, R.; Rotondo, V. y Vinocur, M. 1995. Agroclimatología de Río Cuarto - 1974/1993. Vol. I FAV. UNRC. Río Cuarto. Córdoba. Argentina.

25. Steel, R. y TORRIE, J. 1997. Bioestadística: Principios y Procedimientos. Mcgraw-Hill Segunda Edición. pp. 622.

26. Whittaker, R.; Levin, S. y Root, R. 1973. Niche, Habitat and Ecotope. The American Naturalist, 107: 321-338.

27. Wysiecki, M. 1993. Productividad Primaria Neta Aérea de un Pastizal Natural de la provincia de La Pampa, Argentina. Revista de la Facultad de Agronomía, La Plata Nº9. 